\title{
Revisión crítica del ensayo de cristalización de sales
}

\section{RESUMEN}

Dentro de los ensayos de alterabilidad o de envejecimiento artificial acelerado, el ensayo de cristalización de sales es -por su sencillez y agresividad-uno de los más idóneos para la evaluación de la durabilidad de los materiales rocosos. No obstante, los resultados obtenidos pueden verse condicionados por las diferencias en cuanto al procedimiento experimental.

En este trabajo se revisan las principales variables que intervienen en dicho ensayo, entre las cuales pueden mencionarse: concentración de la solución salina, características de la inmersión, temperatura y tiempo de secado de las muestras, etc.; así como los criterios utilizados en la valoración de los daños generados.

El conocimiento y control de estos factores facilita la optimización de este ensayo, y la correcta interpretación de los resultados obtenidos.

\section{ABSTRACT}

The salt crystallisation test is, for its simplicity and aggressivity, one of the most suitable ageing tests to evaluate the durability of rock materials. However the results of this test could be conditioned by differences in their experimental procedure.

In this article the principal variables involved in the salt crystallisation test are reviewed: concentration of the solution, type of immersion, temperature and time of drying of the specimens, etc.; as well as the criteria used in the evaluation of induced damages.

The knowledge and control of these factors facilitates the optimization of the test, and the correct interpretation of the obtained data.

\section{INTRODUCCION}

Los ensayos de alterabilidad o envejecimiento artificial acelerado se utilizan para evaluar en el laboratorio, de forma rápida y sencilla, la resistencia de los materiales rocosos, así como de otros materiales de construcción (ladrillos, aglomerantes, etc.), a la acción de diversos agentes de alteración: agua, hielo, sales solubles, contaminantes atmosféricos, etc.

Dichos ensayos tienen especial significación para conocer la durabilidad de las diferentes litologías, bien sea de muestras de cantera, o de aquellas otras ubicadas en ambientes específicos (Esbert y Ordaz, 1985). También pueden utilizarse para evaluar la idoneidad de determinados productos de tratamiento, (Laurenzi y Santamaría, 1985).

Diversos ensayos de alterabilidad (humedadsequedad, heladicidad, cristalización de sales, atmósferas contaminadas, radiaciones ultravioletas), han sido ampliamente analizados y comentados en la bibliografía reciente (Price, 1975 y 1978; Marschner, 1978; Gerard 1978 y
1985; etc.). En este trabajo se hace revisión del ensayo de cristalización de sales aplicado a los materiales rocosos.

Como es sabido el ensayo consiste básicamente en la repetición de un número determinado de ciclos, en cada uno de los cuales las muestras son mantenidas durante un cierto tiempo en una solución salina, dejándose secar a continuación, con el fin de que cristalice la sal en su interior.

Ensayos de cristalización de sales se vienen utilizando de antiguo. De hecho, los experimentos de disgregación de materiales rocosos por la acción de las sales arrancan de principios del siglo pasado. Fue en esta época cuando el ingeniero francés Brard ideó el primer método de ensayo a partir de una solución saturada de sulfato sódico, como una vía rápida de reconocimiento del carácter heladizo de los materiales pétreos. Sistematizado el ensayo por Thury (1828), gozó de gran aceptación entre arquitectos e ingenieros y fue ampliamente utilizado, con modificaciones, a lo largo del siglo pasado, extendiéndose su apli- 
cación a otros materiales de construcción (ladrillos y cementos, principalmente).

Sin embargo, la verdadera naturaleza del "ensayo de Brard" y su idoneidad como ensayo de "heladicidad" fue puesta en tela de juicio en más de una ocasión, señalándose como principal objeción que los resultados del mismo venían a probar tan sólo la poderosa acción de sulfato sódico, mucho mayor en realidad que la del hielo (Luquer, 1895).

Ya en el presente siglo, Schaffer (1932), Honeyborne y Harris (1958), y Price (1978) entre otros, estudiaron la funcionalidad y efectividad del ensayo de cristalización de sales. Una documentada revisión histórica acerca de dicho ensayo, en relación con la alteración de las rocas y sus implicaciones, fue dada por Evans (1969).

Actualmente se hallan normalizados algunos ensayos de cristalización de sales para diversas clases de materiales de construcción. Entre estas normas pueden destacarse las de la R.I.L.E.M. (1980) -ensayos V.1a, V.1 b y V.2-, y las D.I.N. - norma 52111- También cabe citar: A.S.T.M. (norma: C 88-76), U.N.E. (norma: 7/136-58) y la del laboratorio del Transporte (norma: NLT 158/72), si bien estas tres últimas han sido elaboradas especialmente para áridos.

Finalmente, es de destacar el papel jugado por las sales solubles en la deterioración de las rocas. En este sentido puede señalarse la evolución de las sales solubles en el perfil de alteración (Iñiguez, 1961), así como los tipos de alteración en función de las sales presentes y su hábito de cristalización (Arnold y Kueng, 1985; Binda et al., 1985).

\section{PREPARACION DE LAS MUESTRAS}

De acuerdo con las características del trabajo y de los materiales estudiados, se determina el número, tamaño y forma de las muestras a ensayar. El número de muestras depende fundamentalmente de la homogeneidad del material $y$, a posteriori, de la dispersión de los resultados obtenidos. Puede considerarse como número mínimo el de tres muestras, por cada tipo homogéneo o variedad objeto de estudio.

El tamaño de las muestras puede estar condicionado por el de sus componentes minerales o texturales, recomendándose, en general, un tamaño diez veces superior a las dimensiones del mayor de ellos. En cuanto a su forma, en todos los casos se utilizan muestras próximas a modelos geométricos (cubos, paralelepipedos, cilindros, discos, etc.). En general, las formas y dimensiones más utilizadas - recomendadas por las normas R.I.L.E.M.- son cubos de $5 \mathrm{~cm}$ de arista.

\section{VARIABLES QUE AFECTAN AL ENSAYO}

Distintas variables o factores influyen en este ensayo, tal como han señalado diferentes autores (Price, 1978; Marschner, 1978; Binda et al., 1985). Estas variables pueden ser agruppadas de la siguiente forma:

- Relativas a la naturaleza y concentración de la sal.

- Relativas a la inmersión: tipo, tiempo y temperatura.

- Relativas al secado: temperatura, tiempo y humedad ambiental.

\subsection{Naturaleza y concentración de la sal}

La sal más utilizada es el sulfato sódico. En menor proporción se utilizan cloruro sódico, sulfato magnésico y sulfato amónico, así como distintas mezclas de sales. Los ensayos con cloruro sódico son empleados para simular la acción agresiva de aerosoles marinos (RossiManaresi, 1976); las mezclas de sales, en situaciones especificas donde se conocen las sales solubles presentes.

Al cristalizar el sulfato sódico presenta, esencialmente, dos fases estables:

- Una fase anhidra, de aspecto pulverulento: thenardita, $y$

- Otra fase decahidratada, que cristaliza en finas agujas: mirabilita.

A temperaturas superiores a $32.4^{\circ} \mathrm{C}$ cristaliza como fase estable la sal anhidra y por debajo la hidratada (Fig. 1). A temperaturas inferiores a la indicada, y en presencia tanto de agua líquida como vapor de agua, la fase anhidra tien-

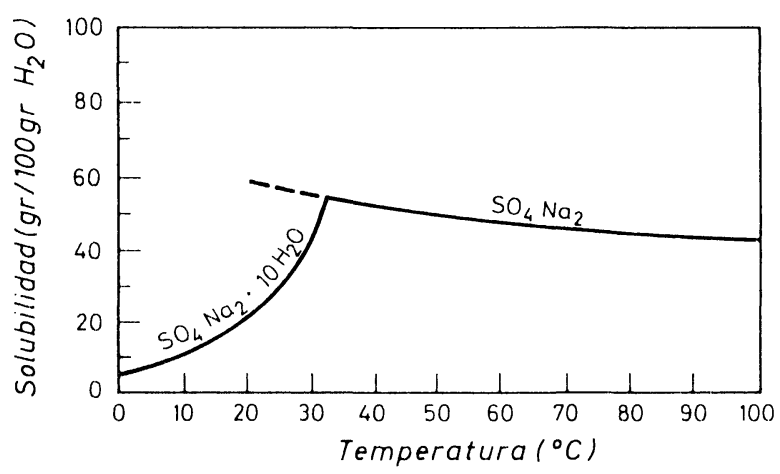

Fig. 1.-Solubilidad del sulfato sódico (expresada en gramos de sal anhidra, por 100 gramos de agua) en función de la temperatura, mostrando el cambio de fase que tiene lugar a $32.4^{\circ} \mathrm{C}$. 
de a hidratarse, si bien dicho proceso puede ser relativamente lento. En la Fig. 2 se observa cómo las oscilaciones diarias experimentadas por la temperatura y humedad relativa del ambiente, pueden afectar dicho equilibrio (Arnold, 1976; Baronio et al., 1983).

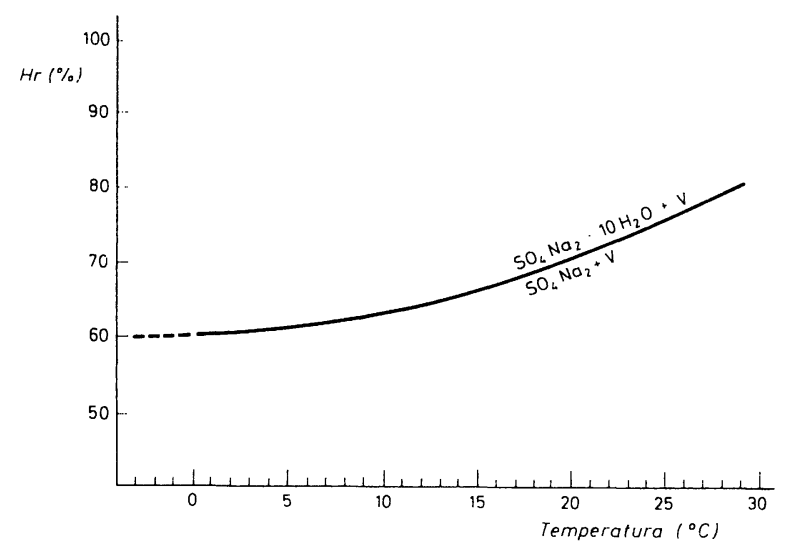

Fig. 2.-Diagrama de fases del sulfato sódico en función de la temperatura y humedad, dentro de condiciones ambientales (Arnold, 1976).

Las concentraciones de solución salina utilizadas varian desde soluciones saturadas (20\% para el $\mathrm{Na}_{2} \mathrm{SO}_{4} .10 \mathrm{H}_{2} \mathrm{O}$ a $20^{\circ} \mathrm{C}$ ) hasta soluciones al $10 \%$. Las normas R.I.L.E.M. recomiendan concentraciones al $14 \%$ (ensayo: V.1) y al $10 \%$ (ensayo: V.2); las normas D.I.N. consideran soluciones saturadas. Price (1975), Niesel (1978) y Leary (1981) entre otros, trabajan con soluciones de sulfato sódico al $14 \%$. Puede señalarse que al disminuir la concentración de la sal disminuyen los daños producidos.

\subsection{Caracteristicas de la inmersión}

Existen dos tipos de inmersión en la solución salina:

- Inmersión total de las muestras. En este caso suelen tomarse unos cinco volúmenes de disolución por volumen de roca.

- Inmersión parcial, con ascenso de la disolución por capilaridad. (De acuerdo con las normas R.I.L.E.M., la parte sumergida para cubos de $5 \mathrm{~cm}$ de lado es de 0.5 a $1 \mathrm{~cm}$ ).

El tiempo de permanencia de las muestras en la disolución varía, según las distintas normas y autores, de 2 a 24 horas. Las normas R.I.L.E.M., así como diversos autores (Price, 1978; Cuttano et al., 1981) consideran suficientes 2 horas; las normas D.I.N. recomiendan 16 horas. Otros autores (Accardo et al. 1978; Zehnder y Arnold, 1984) utilizan tiempos de 24 horas. Price (1978) señala que al aumentar el tiempo de inmersión más allá de las primeras horas, no se observan variaciones significativas en el deterioro producido.

En este sentido pueden señalarse los resultados obtenidos en nuestro laboratorio, donde se ha determinado la ganancia en peso de las muestras tras su inmersión en la solución salina (Fig. 3). Puede observarse cómo a partir de la tercera hora de inmersión el peso permanece prácticamente constate. Por otra parte, la penetración de la solución salina está condicionada por el tamaño de los poros, de manera que a partir de cierto tiempo la pequeña ganancia de peso que pueda existir corresponde a poros de pequeño tamaño, a los que accede agua con muy baja concentración de sales, lo cual justifica la escasa influencia de dicha variable en el deterioro.

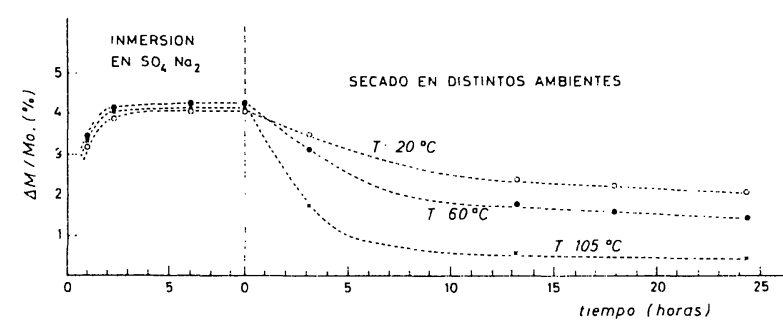

Fig. 3.-Variaciones en el peso de distintas muestras de caliza durante la etapa de inmersión en la solución salina, y durante el secado a distintas temperaturas.

De gran importancia es el control de la temperatura de la solución salina, la cual debe mantenerse baja y constante, puesto que al aumentar ésta disminuyen los daños originados por las sales. Temperaturas superiores a $32.4^{\circ} \mathrm{C}$ impiden la hidratación de la sal, desapareciendo pŕacticamente los daños.

\subsection{Características del secado}

El secado de las muestras es, sin duda, el factor más importante en cuanto a los daños generados por este ensayo, especialmente en lo referente a la temperatura y tiempo de secado.

Las normas R.I.L.E.M. (V.1) y D.I.N., así como algunos autores (Price, 1978), utilizan altas temperaturas de secado: $105^{\circ} \mathrm{C}$. Otro grupo de autores (Marschner, 1978; Accardo et al., 1981; Cuttano el al., 1981), asi como las normas R.I.L.E.M. (V.2), consideran temperaturas más bajas: $60^{\circ} \mathrm{C}$. Estas dos temperaturas son, sin duda, las más utilizadas, estando la última recomendada en presencia de determinados componentes ( $p$. ej. arcillas) o productos de tratamiento, que puedan verse afectados por temperaturas más elevadas. Para tiempos sufi- 
cientemente largos, el secado puede realizarse a temperatura ambiente (Baronio, 1983).

Otra variable a tener en cuenta es el tiempo de secado. Normalmente se emplean tiempos que van desde 4 a 24 horas, mayores cuanto más baja es la temperatura de secado. La mayoría de los autores utilizan tiempos más bien largos; las normas R.I.L.E.M. consideran tiempos de 10 a 20 horas $y$, únicamente, las normas D.I.N. indican 4 horas.

En ensayos llevados a cabo en nuestro laboratorio (Fig. 3), puede observarse cómo el grado de secado de las muestras depende fundamentalmente de la temperatura a la que éste tiene lugar, influyendo de forma muy acusada dicha temperatura sobre la velocidad de secado. Al aumentar la temperatura, la etapa inicial -correspondiente al "secado rápido" (Alonso 1986; Alonso et al. 1987) - es más corta e importante. A partir de un cierto tiempo - unas 10 horas- el secado pasa a depender básicamente de la temperatura, por lo que prolongar el tiempo de secado no presenta interés; contrariamente, pequeñas variaciones de temperatura resultan mucho más significativas.

En dichos ensayos también se pone de manifiesto que la temperatura de secado $-y$ en consecuencia su velocidad- es un factor fundamental en cuanto al poder agresivo del ensayo, disminuyendo éste de forma espectacular para secados lentos a bajas temperaturas (Fig. 4). Cuando el secado se realiza a temperatura ambiente se requieren tiempos mucho mayores, así como un control de la humedad relativa del entorno, ya que de ella depende que la sal cristalice anhidra o hidratada. En cualquier caso los efectos así obtenidos no son comparables a los alcanzados con altas

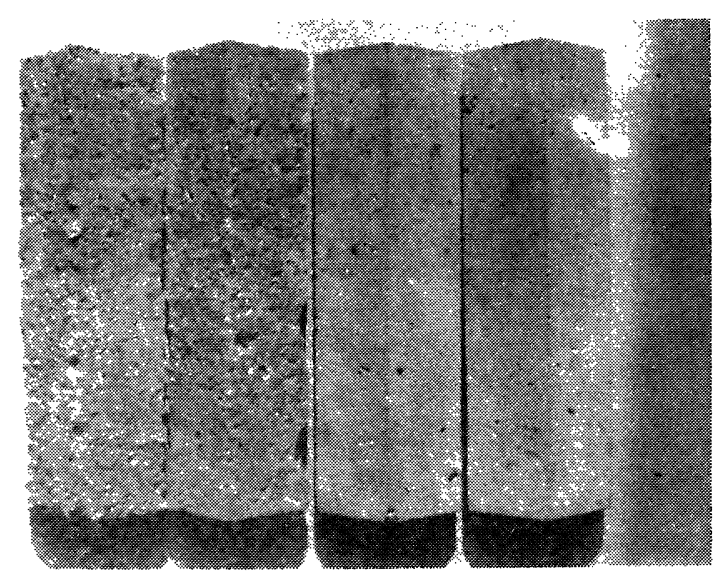

Fig. 4.-Diferencia entre los daños presentados por un mismo tipo rocoso (Caliza del Páramo) cuando es sometido a los mismos ciclos de cristalización de sales, variando únicamente la temperatura de secado. Izq.: Muestra secada en horno (105 $\mathrm{C})$. Der.: Muestra secada a temperatura ambiente $\left(20^{\prime \prime} \mathrm{C}\right)$. temperaturas de secado (Baronio, 1983; Binda y Baronio, 1984).

Otras características importantes del secado son el precalentamiento del horno antes de la introducción de las muestras, así como el mantenimiento de un ambiente húmedo en su interior. Ambos hechos disminuyen la migración de la solución salina hacia la superficie en las etapas iniciales, influyendo en la agresividad del ensayo, en el sentido de acentuar los daños.

Finalmente, antes de comenzar un nuevo ciclo debe asegurarse que las muestras estén en su totalidad a temperatura ambiente. Para los tamaños habituales esto requiere un tiempo minimo de dos horas. Cuando se mantiene durante un tiempo prolongado este enfriamiento de las muestras en ambiente suficientemente húmedo, comienzan los procesos de hidratación de la sal.

\subsection{Número de ciclos}

Se observan dos tendencias en cuanto al número de ciclos a realizar: Un número predeterminado de ciclos, observándose después los daños producidos en las muestras al cabo de los ciclos; o bien, realizar ciclos hasta la degradación total de las muestras, tomándose en este caso como índice de durabilidad el número de ciclos resistidos.

En el primer caso el número de ciclos recomendados por las normas R.I.L.E.M. es de 15, oscilando normalmente entre 10 y 30 en la mayoría de los trabajos consultados. La elección de dicho número está condicionado por las características de los materiales, por lo que a priori no es fácil de establecer.

\section{DAÑOS GENERADOS POR EL ENSAYO}

A partir del primer ciclo, la inmersión de nuevo de las muestras en la solución salina produce la hidratación de la sal presente en los poros, teniendo lugar el deterioro más importante en ese momento - nada más sumergirlas en la disolución- como se corrobora experimentalmente. Binda et al. 1985, atribuyen también parte de los daños a procesos de cristalización e hidratación secundaria, provocados por el agua sobrante, procedente de la deshidratacion de la sal.

La degradación producida por el ensayo es de origen fundamentalmente mecánico, consecuencia de las presiones de hidratación desarrolladas durante el cambio de fase de sal anhidra a hidratada, cuando ésta se encuentra confinada en las reducidas dimensiones de los 
conductos porosos. Dicha transformación comporta un notable incremento de volumen, alrededor del $400 \%$ en el caso de sulfato sódico. La abundancia de microporos (radio $<0,1$ $\mu \mathrm{m})$ parece decisiva respecto a los efectos disruptivos de las sales (Hatsagortsian, 1985).

Los daños dependen fundamentalmente del grado de coherencia y compacidad de los materiales. Así, en rocas suficientemente porosas y coherentes, los poros más próximos a la superficie rompen cuando la resultante de los esfuerzos de tracción ejercidos sobre sus paredes supera su resistencia mecánica (Fig. 5). Como consecuencia, tiene lugar una pérdida contínua de material de la superficie a lo largo del ensayo, con saltación de partículas -en algunas calizas (Alonso, 1986) - y desagregación granular - predominantemente en areniscas (Esbert et al., 1984)-; su alcance depende de las características petrográficas y configuración del sistema poroso de los materiales. Este hecho da lugar al característico aspecto "picado" que presentan algunas muestras en superficie (Fig. 6, superior).
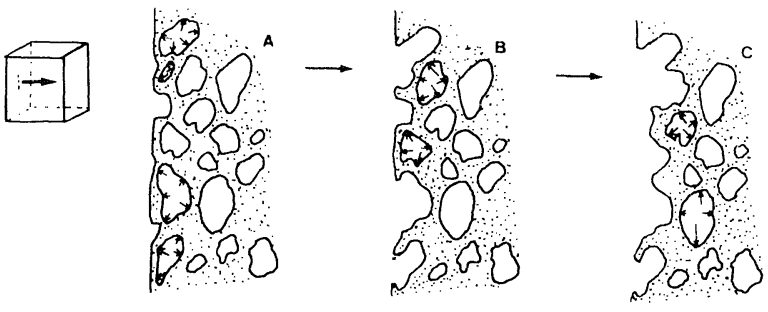

Fig. 5.- Perfiles (A, B, C) donde se muestra la evolución que experimenta una roca porosa, a lo largo de sucesivos ciclos de cristalización de sales, como consecuencia de la pérdida de material de su superficie.

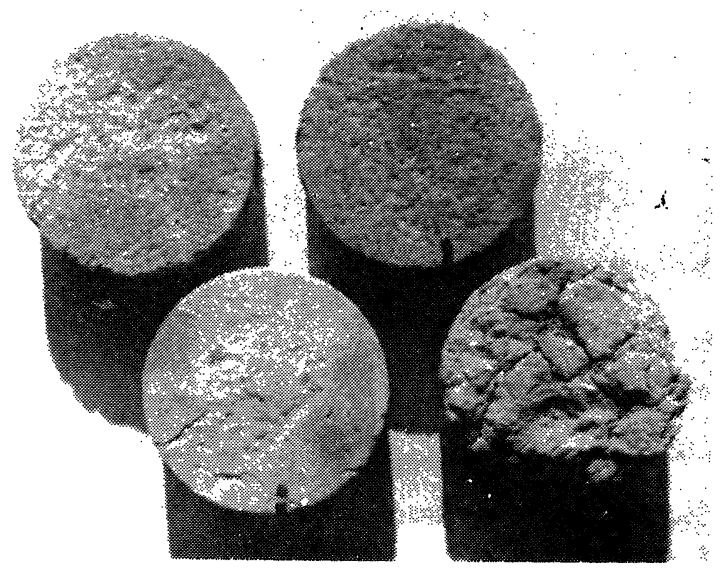

Fig. 6.-Aspecto que presentan diferentes rocas carbonatadas tras la realización de 12 ciclos. En la parte superior puede observarse -en una roca porosa y relativamente coherente (Dolomía de Boñar) - el característico "picado" de la superficie. En la parte inferior -en roca de grano más fino y rica en arcillas (Caliza de Laspra)- se aprecia el "cuarteado" de las muestras, próximo ya a su total desmoronamiento. (Diámetro $35 \mathrm{~mm}$.).
En rocas menos coherentes y de tamaño de grano muy fino -en presencia de suficiente. proporción de arcillas - el deterioro es diferente. Puede observarse en este caso un cuarteado de las muestras, con pérdida de material más pulverulento que, eventualmente, puede conducir a su total fragmentación o desmoronamiento (Fig. 6, inferior).

Cuando el secado de las muestras tiene lugar a temperatura ambiente, la migración más lenta del agua hacia la superficie favorece que ésta vaya acompañada de las sales disueltas. Al producirse la evaporación de dicha agua, se generan abundantes eflorescencias (Fig. 4) disminuyendo de forma drástica los daños presentados por las muestras.

\section{VALORACION DE LOS DAÑOS PRODUCIDOS}

Además de las variables consideradas, referentes al procedimiento experimental a seguir en el ensayo, presenta especial interés la correcta valoración de los daños producidos en las muestras. En este sentido, pueden tenerse en cuenta diversos criterios (Accardo, 1981; Cassaro et al., 1982; Alonso, 1986), sin perder de vista que, para la optimización y normalización de este ensayo, dichos criterios han de ser reducidos en cuanto a número y lo más sencillos y precisos posible.

Entre los diferentes criterios utilizados por distintos autores y normas de ensayo, pueden señalarse los siguientes:

- Observación de las superficies.

- Pérdida de peso de las muestras.

- Variación de propiedades físicas (porosidad, capilaridad, resistencia mecánica, velocidad de propagación de ondas, etc.).

\subsection{Observación de las superficies}

Constituye sin duda el criterio fundamental, a pesar de su carácter cualitativo. Entre los daños observados cabe destacar la fisuración y la pérdida de material, atendiendo en este caso al tamaño de las partículas desprendidas. En estudios detallados esta observación puede ser llevada a cabo bajo microscopía electrónica de barrido (Fig. 7).

La valoración de los daños puede documentarse con la obtención de fotografías. Las normas R.I.L.E.M. recomiendan una valoración relativa, por comparación con muestras cuya resistencia a la intemperie es conocida, así como con la introducción de una muestra de referencia en cada serie de ensayos. Una cierta cuanti- 
ficación se consigue marcando una de las caras de las muestras con tinta indeleble al comienzo del ensayo, y determinando el porcentaje de superficie que se mantiene coloreada después de cada ciclo.

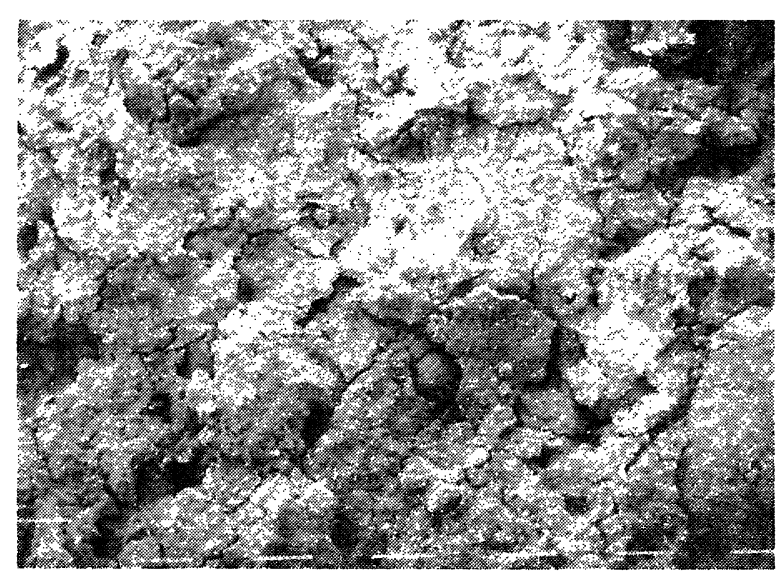

Fig. 7.-Observación en microscopia electrónica de barrido de la superficie de una roca calcárea (Caliza del Páramo), después de realizados 12 ciclos. Puede observarse el aspecto deteriorado de su superficie, y el desprendimiento de material - desconchados y descamaciones- en relación con fisuras subparalelas a dicha superficie ( 1 marcador $=100 \mu \mathrm{m})$.

\subsection{Pérdida de peso de las muestras}

Este parámetro es, sin duda, el más utilizado; no obstante, debe señalarse que no todos los materiales presentan el mismo tipo de deterioro, por lo que es aconsejable que dichos valores se acompañen de la descripción de los daños observados.

La pérdida de peso de las muestras se expresa en forma de porcentaje respecto a su peso seco inicial. Dichas determinaciones pueden realizarse a lo largo de los ciclos, efectuándose normalmente antes de su comienzo. Como consecuencia de la introducción de sal en los poros, en los primeros ciclos se observa cierto

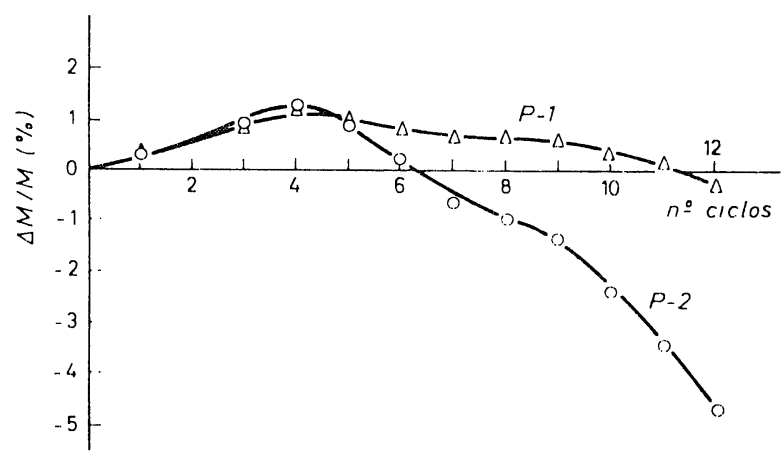

Fig. 8.-Pérdidas de peso experimentadas por dos variedades calcáreas (Caliza del Páramo: P-1 y P-2). incremento en el peso de las muestras (Fig. 8 y 9). Finalizando el ensayo, suele procederse al lavado de las sales con renovación periódica del agua de lavado, determinándose entonces la pérdida neta de peso de las muestras, correspondientes al número total de ciclos efectuados.

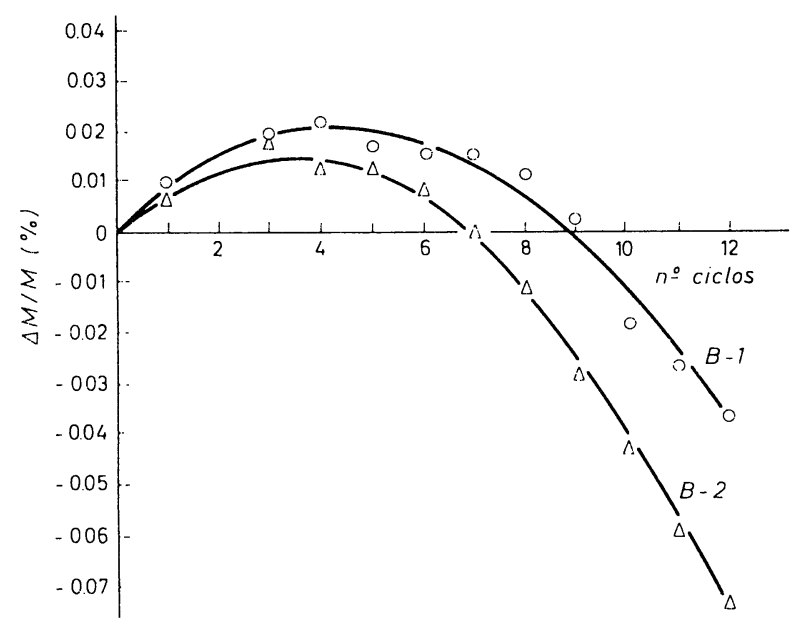

Fig. 9.-Pérdidas de peso correspondiente a dos variedades de dolomía cristalina (Dolomía de Boñar: B-1 y $\mathrm{B}-2)$.

\subsection{Variación de propiedades físicas}

La determinación de ciertas propiedades físicas de los materiales (p. ej. porosidad, capilaridad, resistencia mecánica y velocidad de ondas elásticas), antes y después de realizar el ensayo, constituye otro factor en la valoración y cuantificación de los daños producidos.

La variación de la porosidad abierta o efectiva resulta por sí misma un parámetro poco significativo. En rocas cristalinas, sin embargo, donde no se aprecian daños en superficie, ni pérdidas de peso, las pequeñas variaciones de porosidad constituyen un buen índice de microfisuración interna (Alonso, 1986). Lo mismo puede decirse de la absorción de agua por capilaridad.

Las variaciones de propiedades mecánicas y elásticas son, a veces, tenidas en cuenta en la evaluación de los daños. Accardo et al. (1981) determinan la resistencia a la compresión y el módulo de Young en mármoles sometidos hasta 30 ciclos, no encontrando diferencias apreciables en los primeros ciclos. Alonso (1986), para distintos tipos de calizas y 12 ciclos, no observa diferencias de resistencia a la tracción entre discos intactos y ensayados.

También se emplea la velocidad de propagación de ondas elásticas, realizándose determinaciones antes y después del ensayo. En 
este caso además, dado que se trata de un ensayo no destructivo, puede ser utilizado a lo largo de los ciclos. Este parámetro presenta gran interés como índice de alteración o de calidad, especialmente en rocas cristalinas (Tourenq et al., 1971; Accardo et al., 1981).

\section{CONCLUSIONES}

El ensayo de cristalizacion de sales constituye un procedimiento experimental rápido y sencillo para evaluar la durabilidad de los materiales rocosos. Los daños generados por el ensayo deben ser considerados fundamentalmente en términos relativos, resultando difícil su correlación con tiempos de exposición de los materiales en ambientes naturales.

No existe un probado paralelismo entre este ensayo y el de heladicidad, por lo que no debe considerarse como un ensayo acelerado de resistencia al hielo, sino más bien como un valor independiente en la evaluación de la durabilidad de las rocas.

El ensayo presenta interés tanto en la valoración de la calidad de los materiales, como en la de los tratamientos a que éstos pueden verse sometidos, permitiendo la obtención de índices de calidad a partir de las variaciones experimentadas por los diferentes parámetros físicos.

De acuerdo con lo expuesto acerca de las variables que intervienen en el ensayo, así como de los criterios utilizados en la valoración de los daños -y desde un punto de vista eminentemente práctico- pueden señalarse algunas observaciones relativas al procedimiento de ensayo:

- Para el ensayo con sulfato sódico decahidratado, es aconsejable preparar solucio- nes acuosas al $14 \%$, tomándose cinco volúmenes de solución por cada volumen de roca y renovándose cada dos ciclos.

- La duración más conveniente de cada ciclo es de 24 horas. De acuerdo con esto, pueden considerarse en cada uno de ellos las siguientes etapas:

- Inmersión total de las muestras en la solución a $20^{\circ} \mathrm{C}$ durante 4 horas.

- Secado de las muestras en un horno, previamente calentado a $60^{\circ} \mathrm{C}$ o bien a $105^{\circ} \mathrm{C}$-según el tipo de material a ensayar-, manteniéndose en dichas condiciones durante 14 horas.

- Enfriado a $20^{\circ} \mathrm{C}$ durante otras 6 horas, en ambiente con humedad relativa baja y constante.

- El número mínimo de ciclos a realizar se cifra en diez.

La valoración de los resultados debe atender, al menos, a los daños observados en superficie y a la pérdida de peso de las muestras, teniendo en cuenta que estos últimos valores sólo son comparables, en principio, entre muestras rocosas del mismo tipo.

\section{AGRADECIMIENTOS}

A la Comisión Asesora de Investigación Científica y Técnica (Proyecto N. 2573/83), por el soporte prestado a estos estudios. A la Fundación Ramón Areces, que con la financiación del Proyecto "Evaluación del estado de alteracion y alterabilidad de los materiales rocosos de las Catedrales de León y Sevilla", ha permitido asimismo profundizar en el conocimiento de la alteración que experimentan las rocas.

\section{BIBLIOGRAFIA}

ACCARDO, A., MASSA, M., ROSSI-DORIA, P. y TABASSO, M., (1978): Measures of porosity and of mechanical resistance in order to evaluate the state of deterioration of some stones. Proc. of the Int. Symp. on Deterioration and Protection of Stone Monuments, París, 2.1, 22 pp.

ACCARDO, G., MASSA, S., ROSSI-DORIA, P., SAMMURI, P. y TABASSO, M., (1981): Artificial weathering of Carrara marble. Relationships between the induced variations of some physical properties. The Conservation of Stone II, R. Rossi-Manaresi (Ed.), Proc. of the Int. Symp., Bologna, pp. 243-273.

ALONSO RODRIGUEZ, F. J., (1986): Caracterización petrofísica y alterabilidad de calizas y dolomias. Tesis Doctoral, Facultad de Geología. Universidad de Oviedo, 309 p.

ALONSO, F. J., ESBERT, R. M. y ORDAZ, J., (1987): Comportamiento hídrico de calizas y dolomías. Bol. Geol. Min. (en prensa).

ARNOLD, A., (1976): Soluble salts and stone weatherning. The Conservation of Stone I, R. Rossi-Manaresi (Ed.), Proc. Int. Symp., Bologna, pp. 133-135.

ARNOLD, A., (1981): Nature and reactions of saline minerals in walls. The Conservation of Stone II, R. Rossi-Manaresi (Ed.), Proc. Int. Symp., Bologna, pp. 13-23

ARNOLD, A. y KUENG, A., (1985): Crystallization and habits of salt efflorescences on walls I: Methods of investigation and habits. $V$ Int. Cong. on Deterioration and Conservation of Stone, Vol. 1, Lausanne, pp. 255-267. 
BARONIO, G., BINDA, L. y SCIROCCO, F. (1983): Accelerated aging and models for the progressive chemico-physical deterioration of bricks in the diagnosis of masonry structures. Material Science and Restoration, F. H. Wittman (Ed.), Proc. Int. Coll., Edition Lack and Chemie, pp. 391-395.

BINDA, L. y BARONIO, G., (1984): Measurement of the resistance to deterioration of old and new bricks by means of accelerated aging test. Durability of Building Materials, 2 (2), pp. 139-154.

BINDA, L., BARONIO, G. y CHAROLA, A. E., (1985): Deterioration of porous materials due to salt crystallization under different thermohigrometric conditions. V. Int. Cong. on Deterioration and Conservation of Stone, Vol. 1, Lausanne, pp. 279-289.

CASSARO, M. A., GAURI, K. L., SHARIFINASSAB, M. y SHARIFIAN, A., (1982): On the strength and deformation properties of Indiana Limestone and concrete in the presence of salts. IV Int. Cong. on the Deterioration and Preservation of Stone Objects, Kentucky, pp. 57-76.

CUTTANO, M., MASTRONARDI, P. y ROSSI-MANARESI, R., (1981): Alveolar weathering of the "tuff" of Matera mechanism of deterioration and effectiveness of preservation treatments. The Conservation of Stone II, R. Rossi-Manaresi (Ed.), Proc. of the Int. Symp., Bologna, pp. 355-377.

ESBERT, R. M. y ORDAZ, J., (1985): Alterabilidad de la piedra monumental de Laspra (Asturias). Trabajos de Geología, Univ. de Oviedo, 15, pp. 325-331.

ESBERT, R. M., ORDAZ, J., ALONSO, F. J. y VALDEON, L., (1984): Influencia de las características petrográficas en la durabilidad de areniscas utilizadas en monumentos. I Cong. Español de Geología, Segovia, T. II, pp. 921-931.

ESBERT, R. M., ORDAZ, J. y VALDEON, L., (1985): Relaciones entre porosidad, contenido en arcillas y durabilidad de areniscas. Materiales de Construcción, 200, pp. 15-22.

EVANS, I., (1969): Salt crystallization and rock wheathering: a review. Revue de Géomorphologie Dynamique, Année XIX, N. 4, pp. 153-177

GERARD, R., (1978): Trois méthodes d'éssais de géliveté récemment normalisées en Belgique. Proc. of the Int. Symp. on Deterioration and Protection of Stone Monuments, París, 3.2, 18 pp.

GERARD, R., (1985): Détermination de la durabilité au gel par un méthode de capillarité. V Int. Cong. on Deterioration and Conservation of Stone, Lausanne, Vol. 1, pp. 157-166.

HATSAGORTSIAN, Z., (1985): Principes expérimentaux et théoriques pour l'évaluation de durabilité de la pierre. $V$ Int. Cong. on Deterioration and Conservation of Stone, Lausanne, Vol. 1., pp. 195-202.

HONEYBORNE D. B. y HARRIS, P. B., (1958): The estructure of porous building stone and its relations to its chemical behaviour. Structures and properties of porous materials, Everett D. H. (Ed.), Butterworth's, London, pp. $343-363$.

IÑIGUEZ HERRERO, J., (1961): Alteración de calizas y areniscas como materiales de construcción. Publ. del Ministerio de Educación, Dir. Gen. Bellas Artes, Madrid, 147 p.

LAURENZI TABASSO, M. y SANTAMARIA, U., (1985): Consolidant and protective effects of different products on Lecce Limestone. V Int. Cong. on Deterioration and Conservation of Stone, Vol. 2, Laussanne, pp. 697-707.

LEARY, E., (1981): A preliminary assessment of capillarity tests as indicators of the durability of British limestones. The Conservation of Stone II, R. Rossi-Manaresi (Ed.), Proc. of the Int. Symp., Bologna, pp. 73-90.

LUQUER, L., (1895): The relative effects of frost and the sulphate of soda efflorescence test of building stones. Amer. Soc. Civil Engineers. Trans. 33, pp. 235-26.

MARSCHNER, H., (1978): Application of salt crystallisation test to impregnated stones. Proc. of Int. Symp. on Deterioration and Protection of Stone Monuments, Paris, 3.4, $16 \mathrm{pp}$.

NIESEL, K., (1978): L'évolution de la diminution de la masse comme base pour l'évaluation de l'éssai de cristalisation. Proc. of Int. Symp. on Deterioration and Protection of Stone Monuments, París, 3.5, $13 \mathrm{pp}$.

PRICE, C. A., (1975): Testing porous building stone. Architects Journal, 162, pp. 337-339.

PRICE, C. A., (1978): The use of the sodium sulphate crystallisation test for determining the weathering resistance of untreated stone. Proc. Int. Symp. on Deterioration and Protection of Stone Monuments, Paris, 3.6, 23 pp.

ROSSI-MANARESI, R., (1976): Treatments for sandstone consolidation. The Conservation of Stone I, R. Rossi-Manaresi (Ed.), Proc. of the Int. Symp., Bologne, pp. 547-571.

R.I.L.E.M., (1980): Essais recommandés pour mesurer l'altération des pierres et évaluer l'eficacité des méthodes de traitements. Matériaux et Constructions, Bull. RILEM, 13 (75), pp. 216-220.

SCHAFFER, R., (1932): The wheathering of natural building stones. Dpt. Sci. Ind. Res., Building Res., Special Rept. 18, London, $149 \mathrm{pp}$

THURY, H. et al., (1828): Sur le procédé proposé par $M$. Brard pour reconnaître immédiatement les pierres qui ne pouvent pas résister à la gelée. Ann. de Chimie et de Physique, 38, pp. 160-192.

ZEHNDER, K. y ARNOLD, A., (1984): Stone damage due to formate salts. Studies in Conservation, 29, pp. 32-34. 